\title{
3 Research Square

\section{Changes and clinical significance of invariant natural killer T cells and their subsets in newly diagnosed type 1 diabetic patients}

Hui Guo ( $\nabla$ ghui@jlu.edu.cn )

Jilin University First Hospital

Shuci Tian

Jilin University First Hospital

Lichao Gao

Jilin University First Hospital

\section{Research Article}

Keywords: Invariant natural killer T cell, Diabetes mellitus, Type 1 diabetes mellitus

Posted Date: September 16th, 2021

DOl: https://doi.org/10.21203/rs.3.rs-902038/v1

License: (c) (i) This work is licensed under a Creative Commons Attribution 4.0 International License.

Read Full License 


\section{Abstract}

Objective:The aim of this study was to investigate the changes and clinical significance of the invariant natural killer T(iNKT) cells and their subsets in peripheral blood of newly diagnosed type 1 diabetic patients.

Methods: 23 newly diagnosed type 1 diabetic patients and 17 healthy volunteers(from 2015 to 2019)were collected. Peripheral blood mononuclear cells (PBMC) were isolated by FICOII method, the ratio of main cell subsets of PBMC was determined by Flow cytometry. The changes of iNKT cells and their subsets $(\mathrm{CD} 4+, \mathrm{CD} 4-\mathrm{CD} 8-, \mathrm{CD} 8+, \mathrm{CD} 4+\mathrm{CD} 8+)$ in peripheral blood as well as their correlation with clinical indexes were monitored.

Results: Compared with the healthy group, the absolute TCRV $\beta 11+i N K T$ cell number in the peripheral blood of newly diagnosed type 1 diabetic patients $(p=0.03)$ was significantly higher. The absolute CD4+TCRVß11iNKT cell number $(p=0.00), C D 4+T C R V a 24+i N K T$ cells $(p=0.01), C D 4+T C R V a 24 J 18$ $+i N K T$ cells $(p=0.00), C D 8+T C R V \beta 11+i N K T$ cells $(p=0.021)$ and CD4+CD8+ TCRVa24+iNKT cells $(p=0.002)$ were significantly increased. The absolute CD4-CD8-TCRVa24+iNKT cell number $(p=0.030)$ in T1DM patients was significantly lower than that in HC. In newly diagnosed type 1 diabetic patients, the absolute CD4+TCRV $\beta 11+\mathrm{iNKT}$ cell number was negatively correlated with BMI $(p=0.001, r=-0.492)$, and the absolute CD4+TCRVa24+iNKT cell number was negatively correlated with BMI ( $p=0.014, r=-0.387)$, the absolute CD4+TCRVa24J18+iNKT cell number was negatively correlated with BMI $(p=0.010, r=-0.402)$ and positively correlated with glycated hemoglobin $(P=0.048, r=0.417)$. The absolute CD4+CD8+TCRVa24+iNKT cell number was positively correlated with 30 minutes and 1 hour postprandial C-peptide $(p=0.002, r=0.664 ; p=0.002, r=0.669)$.

Conclusion: Our data indicated a higher absolute iNKT cell number in T1DM patients compared with that in healthy patients, and was accompanied by changes in the number of cell subsets. The immune dysfunction mediated by iNKT cells may associate with the development of type 1 diabetes.

\section{Introduction}

Natural killer T (NKT) cells are specific lymphocytes that mediate innate and acquired immune responses and may participate in complex autoimmune processes. They can kill the target cells directly and interact with antigen-presenting cells and T cells, displaying both T cells and NK cells ${ }^{[1]}$. According to the widely accepted nomenclature, iNKT cells are classified into type I, II, and III NKT cells, each with different functional properties. Type I NKT cells, also known as invariant natural killer T (iNKT) cell, is the most widely studied type of NKT cells. A majority of the NKT cells express an invariant T cell receptor (TCR), consisting of Va24-Ja18 a-chain (Va14-Ja18) in mice ${ }^{[1-5]}$.

The iNKT cells develop in the thymus; most of the cells remain in the thymus after maturation, and the remaining cells migrate to the peripheral immune organs. iNKT cells are abundant in the spleen, liver, thymus, and bone marrow, lymph nodes of the intestine and lungs, peripheral blood, adipose tissue, skin, 
and mucosal surfaces ${ }^{[5,6]}$. Fewer iNKT cells are detected in most organs in humans than those in mice; also, the content of iNKT cells varies but the reason is yet unclear ${ }^{[5]}$.

According to the development of iNKT cells in the thymus and the expression of transcription factors as well as the production of cytokines, type I NKT cells are divided into iNKT-1, iNKT-2, iNKT-17, iNKT-10, and iNKTFH (follicular helper) cells ${ }^{[2]}$. iNKT-1 cells mainly secrete interferon-gamma (IFN- $y$ ), iNKT-2 cells mainly secrete IL-4 and IL-13 ${ }^{[7]}$, iNKT17 mainly secrete IL- 17 , iNKTFH mainly secretes IL-2 $1^{[1]}$, and iNKT10 cells secrete IL-10 ${ }^{[2,3]}$. Based on the expression of CD4 and CD8 molecules, four subpopulations of human iNKT cells have been categorized: CD4+, CD4-, CD4-CD8- (double-negative), and CD4 + CD8+

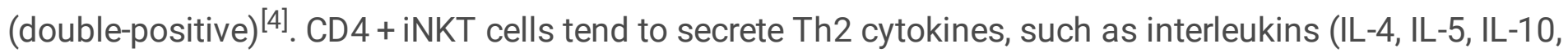
and IL-13), while CD4-CD8- iNKT cells secrete Th1 cytokines, such as tumor necrosis factor (TNF) and IFN-Y.

The glycolipid antigen recognized by iNKT cells is a-galactose acyl sphingosine (a-GalCer) ${ }^{[8]}$, which was originally extracted from spongy cells. a-GalCer activates iNKT cells in mice and humans, presented by CD1d molecules similar to MHC-I. When activated by a-GalCer, iNKT cells produce various cytokines, including IL-4, IFN- $\gamma$, IL-13, and IL-17. These cytokines exert a cytotoxic effect and trans-activates other immune cells, such as NKT and B cells, and play an immunoregulatory role. It has been speculated that iNKT cells have anti-tumor, anti-infection, anti-rejection of organ transplantation effects, and control of autoimmune diseases. Currently, the role of iNKT cells in the development of diabetes is emerging.

Non-obese diabetic (NOD) mice have a tendency to develop autoimmune diabetes spontaneously. Islet autoantigen recognized specifically by T cells and caused an impairment of islet cells in NOD mice. Thus, this animal model is widely applied to the study of the prognosis of type 1 diabetes ${ }^{[9]}$. It is widely recognized that iNKT cell deficiency leads to the development of type 1 diabetes mellitus (T1DM) and a decreased number and the function of iNKT cells in the thymus and spleen of NOD mice. In contrast, the function of the remaining iNKT cells was defective. In addition, the stimulation of the remaining iNKT cells by a-GalCer or transfer of thymic-derived iNKT cells protected against diabetes ${ }^{[10-11]}$. When a-GalCer was applied to NOD mice, cyclophosphamide accelerated the progression of diabetes, while iNKT cells protected against diabetes ${ }^{[12]}$.

High levels of IL-4 and IL-10 were detected when NOD mice were treated with a-GalCer, and the activation of cytokines may be the key to the protective effect of iNKT cells on diabetes ${ }^{[13,14]}$. Furthermore, some studies found that iNKT cells exert significant protection in the early stages of the disease and exhibit age-and dose-dependent effects ${ }^{[15]}$. Several studies have attributed the immunoregulatory function of iNKT cells to the production of cytokines, including IL-2, IL-17, IL-4, IL-10, IL-13, IFN-y, and TNF-a ${ }^{[16,17]}$.

Although the decline in the number of iNKT cells and their protective effect on T1DM has been demonstrated in NOD mice, researchers deduced inconsistent results about the expression and the role of iNKT cells in type 1 diabetic patients. Oikawa et al. ${ }^{[18]}$ found abundant iNKT cells in newly diagnosed type 
1 diabetic patients, whereas in the study of 43 type 1 diabetic patients admitted to Ruijin Hospital ${ }^{[19]}$, Minglan et al. observed a decrease in the number and function of iNKT cells in type 1 diabetic patients. Some other studies suggested no significant difference in the frequency of iNKT cells among patients

with type 1 diabetes ${ }^{[20-22]}$ and no significant effect on the duration of the disease ${ }^{[23]}$. The complexity and contradiction of the number and function in iNKT cells necessitate further exploration. Thus, the present study aimed to investigate the changes and clinical significance of iNKT cells and their subsets in the peripheral blood of newly diagnosed type 1 diabetic patients.

\section{Materials And Methods}

\subsection{Study objects}

A total of 23 patients with newly diagnosed type 1 diabetes were recruited from the inpatient service of the First Hospital of Jilin University in Changchun China, another 17 age and gender matched healthy controls $(\mathrm{HC})$ were recruited from the Physical Examination Center of the same hospital. There were 16 males and 7 females in T1DM group, with an average age of 37.87 years old, while there were 9 males and 8 females in the healthy group with an average age of 44.88 years. Written informed consent was obtained from individual participants and the experimental protocol was approved by the Institute Review Board of the First Hospital of Jilin University.

All patients were initially diagnosed in our hospital or had not been treated after diagnosis. The diagnosis was in accordance with the World Health Organization (WHO) diagnostic criteria for diabetes in 1999: (1) Diabetes symptoms with random blood glucose $\geq 11.1 \mathrm{mmol} / \mathrm{L}$; (2) Fasting blood glucose $\geq 7.0 \mathrm{mmol} / \mathrm{L}$; Oral glucose tolerance test(OGTT) $2 \mathrm{~h}$ blood glucose $\geq 11.1 \mathrm{mmol} / \mathrm{L}$. At the same time, the patients fulfilled one of the following diagnostic criteria for type 1 diabetes: (1) fulminant type 1 diabetes mellitus (FT1DM): diabetic ketoacidosis or ketosis occurred 1 week after the onset of hyperglycemia; (2) blood glucose was $16 \mathrm{mmol} / \mathrm{L}$ at the initial visit, $\mathrm{HbA} 1 \mathrm{C}<8.7 \%$; (3) the function of pancreatic islets coincided with one of the two conditions: fasting C-peptide $<0.3 \mathrm{ng} / \mathrm{mL}$ and post-prandial C-peptide $<0.5 \mathrm{ng} / \mathrm{mL}$. Acute type 1 diabetes mellitus (AT1DM): (1) diabetic ketoacidosis or ketosis occurred within 3 months of the onset of hyperglycemia; (2) insulin treatment is required after diagnosis; (3) glutamic acid decarboxylase antibody (GADA) is positive or fasting C-peptide $<0.6 \mathrm{ng} / \mathrm{mL}$ if GADA is negative.

\subsection{Flow cytometry analysis}

Individual venous blood samples $(10 \mathrm{~mL})$ were collected from each subject, and plasma was collected by centrifugation at $1700 \mathrm{rpm}$ for $15 \mathrm{~min}$ at room temperature. The plasma was discarded, and the remaining blood was diluted with an equivalent volume of normal saline and mixed with a separation medium. The mixed blood cells in a volume ratio of the mixture to the separator, 1:1, were subjected to centrifugation. The first layer was plasma, the second was PBMCs, the third was liquid separation layer, and the fourth was red blood cells. The second layer was collected and mixed with $10 \mathrm{~mL}$ phosphatebuffered saline (PBS), followed by centrifugation at $1700 \mathrm{rpm}$ for $15 \mathrm{~min}$ at room temperature. The 
supernatant was clarified by centrifugation twice, and then, RPMI 1640 was added. The isolated cell suspension of $2 \mu \mathrm{L}$ was mixed with $198 \mathrm{~mL}$ of $0.9 \%$ sodium chloride. Finally, the $1.0 \times 10^{6} / \mathrm{mL}$ cells were counted under a microscope.

The cell suspension of $1 \times 10^{6} \mathrm{cell} / \mathrm{mL}$ was mixed with mouse anti-human CD4 antibody, mouse antihuman CD8 antibody, mouse anti-human CD19 antibody, mouse anti-human CD3 antibody, anti-iNKTPE (TCRV 24 chain antibody, TCRV 11 chain antibody, TCRV 24J 18 chain antibody), respectively for 25 minutes at $4^{\circ} \mathrm{C}$. Subsequently, the cells were washed with PBS at $1200 \mathrm{rpm}$ for $5 \mathrm{~min}$ and stored in PBS at $4^{\circ} \mathrm{C}$ in the dark.

FACS Aria II flow cytometry was used for detection, and the frequency of TCRV $\beta 11+\mathrm{iNKT}$ cells, TCRVa24 + iNKT cells, TCRVa24J18 + iNKT cells, and their subsets (CD4+, CD4-CD8-, CD8+, CD4 + CD8+) was determined by FlowJo (v7.6) software.

\section{Results}

\subsection{The demographic and clinical characteristics of subjects}

A total 23 patients with new diagnosed T1DM and $17 \mathrm{HC}$ were recruited. Their demographic and clinical characteristics are shown in Table 3.1. There were no significant differences distribution of in age, sex, total cholesterol, triglyceride, high-density lipoprotein cholesterol, low-density lipoprotein, WBC and lymphocyte between the two groups $(p>0.05)$. The glycated hemoglobin $(p=0.00)$ and fasting blood glucose $(p=0.00)$ in T1DM group were significantly higher than those in HC group. The fasting $C$ peptide $(p=0.00)$ and BMI $(P=0.002)$ in T1DM group were significantly lower than those in HC group. 
Table 3.1

demographic and clinical characteristics of HC group and T1DM group

\begin{tabular}{|lll|}
\hline & HC Group & T1DM Group \\
\hline age(year) & $43.35 \pm 13.72$ & $37.87 \pm 17.56$ \\
\hline sex(male/female) & $9 / 8$ & $16 / 7$ \\
\hline BMI(Kg/m2) & $23.34 \pm 3.29$ & $19.67 \pm 5.71$ \\
\hline HbA1C(\%) & $4.9 \pm 0.46$ & $13.53 \pm 1.69(9.90-16.90)$ \\
\hline FBG(mmol/L) & $4.98 \pm 0.69$ & $15.05 \pm 4.96(7.49-30.3)$ \\
\hline Fasting C peptide(ng/ml) & $0.99 \pm 0.16$ & $0.35 \pm 0.20(0.1-0.72)$ \\
\hline TCHOL(mmol/L) & $4.98 \pm 0.60$ & $5.16(4.62-5.52)$ \\
\hline TG(mmol/L) & $1.14(0.88-2.18)$ & $1.12(0.78-1.55)$ \\
\hline HDL-C(mmol/L) & $1.42 \pm 0.47$ & $1.22 \pm 0.58$ \\
\hline LDL-C(mmol/L) & $2.85(2.11-2.96)$ & $2.87 \pm 1.21$ \\
\hline WBC(109/L) & $5.67(5.38-7.07)$ & $6.00(4.77-7.51)$ \\
\hline LYMC(109/L) & $2.05 \pm 0.39$ & $2.11 \pm 1.21$ \\
\hline $\begin{array}{l}\text { Note: The measurement data conforming to normal distribution was expressed by mean } \pm \text { standard } \\
\text { deviation. The measurement data of non-normal distribution are described by median and quartile. }\end{array}$ \\
\hline
\end{tabular}

3.2 Changes of absolute iNKT cell number and its subpopulations in healthy group and type 1 diabetes mellitus group

The forward scattering light (FSC) and lateral scattering light (SSC) of peripheral blood cells were detected in HC group and T1DM group.

Circle the lymphocyte at the scattering point, circle the CD3 + CD19-T cell gate, CD4+, CD8+, CD4-CD8- and CD4 + CD8 + cells were labeled with VB110Va24■Va24J18 respectively. As shown in Fig. 3.2.

\subsubsection{Changes of absolute iNKT cell number in healty group and type 1 diabetes mellitus group}

The results of flow cytometry showed that the absolute TCRV $\beta 11+\mathrm{iNKT}$ cell number in peripheral blood of T1DM group was significantly higher than that of HC Group $(p=0.03)$. there were no significance between T1DM group and HC group in the absolute TCRVa $24+$ iNKT cell number and absolute TCRVa24 $\mathrm{J} 18+\mathrm{iNKT}$ cell number $(\mathrm{p}>0.05)$. As shown in Fig. 3.2.1 
3.2.2 Changes of absolute number of subpopulations of iNKT cells in healty group and type 1 diabetes mellitus group

Compared with the healthy group, the absolute numbers of CD4 + TCRV $\beta 11$ iNKT cells $(p=0.00), C D 4+$ TCRVa24 + iNKT cells $(p=0.01)$, CD $4+$ TCRVa24J18 + iNKT cells $(p=0.00)$, CD $8+$ TCRV $111+i N K T$ cells $(p=0.021)$ and CD $4+C D 8+$ TCRVa24 + iNKT cells $(p=0.002)$ in T1DM patients were significantly increased. The absolute number of CD4-CD8-TCRVa24 + iNKT cells $(p=0.030)$ in T1DM patients was significantly lower than that in HC. There were no significantly difference between the two groups in the absolute numbers of CD4-CD8-TCRV $311+$ iNKT cells,CD4-CD8-TCRVa24Ja18 + iNKT cells, CD4 + CD8 + TCRV $311+i N K T$ cells, CD4 + CD8 + TCRVa24Ja18 + iNKT cells CD8 + TCRVa24 + iNKT cells and CD8 + CRVa24Ja18 + iNKT cells. As shown in Fig. 3.2.2

\subsection{Correlation between iNKT cells and clinical indicators}

SPSS software was used for statistical analysis. Pearson test was applied to correlation anaylsis of the measurement data which conform to normal distribution, and Spearman test was applied to correlation anaylsis of the measurement data which do not conform to normal distribution and other types of data. In newly diagnosed type 1 diabetic patients, the absolute CD $4+T C R V \beta 11+i N K T$ cell number was negatively correlated with BMI $(p=0.001, r=-0.492)$, and the absolute CD $4+$ TCRVa24 + iNKT cell number was negatively correlated with BMI $(p=0.014, r=-0.387)$ The absolute CD $4+$ TCRVa24Ja18 + iNKT cell number was negatively correlated with BMI $(p=0.010, r=-0.402)$ and positively correlated with glycated hemoglobin $(p=0.048, r=0.417)$. The absolute CD $4+C D 8+T C R V a 24+i N K T$ cell number was positively correlated with 30 and 60 minutes postprandial C-peptide $(p=0.002, r=0.664 ; p=0.002, r=0.669)$. As shown in Fig. 3.2.2

\section{Discussion}

Type 1 diabetes is defined as diabetes mellitus caused by the destruction of islet cells and absolute deficiency of insulin. The pathogenesis of type 1 diabetes is not yet clarified and is considered to be an autoimmune disease caused by the interaction of genetic and immune factors ${ }^{[24]}$. Accumulating evidence suggests that T cells, NKT cells, and other immune cells contribute to type 1 diabetes development. The immune cells involved in the pathogenesis of type 1 diabetes and the role of iNKT cells is gradually emerging.

NKT cells are specific lymphocytes that mediate innate and acquired immune responses and may participate in complex autoimmune processes. iNKT cells are classified into type I, II, and III NKT cells, each with different functional properties. Type I NKT cells, also known as the invariant natural killer cell, is the most widely studied type of NKT cells. The activated iNKT cells secrete various cytokines, including IL-4, IL-13, IL-17, and IFN-ץ ${ }^{[25]}$. These cytokines exert a cytotoxic effect and also transactivate other immune cells, such as NKT and B cells, thereby exerting an immunoregulatory role. Furthermore, iNKT cells are speculated to play a critical role in anti-tumor, anti-infection, anti-rejection of organ 
transplantation, and control of autoimmune diseases. iNKT cells may play a crucial role in the occurrence and development of T1DM.

We studied the changes of the invariant NKT cells and their subsets in the peripheral blood of newly diagnosed type 1 diabetic patients. The absolute number of TCRV $\beta 11+$ iNKT cells in the peripheral blood of T1DM group was significantly higher than that of HC group $(p=0.03)$. Compared to the healthy group, the absolute number of CD4 + TCRV $\beta 11$ iNKT cells $(p=0.00), C D 4+$ TCRVa24 + iNKT cells $(p=0.01), C D 4$ + TCRVa24J18 + iNKT cells $(p<0.001$. $)$, CD $8+$ TCRV $311+i N K T$ cells $(p=0.021)$, and CD $4+$ CD8 + TCRVa24 + iNKT cells $(p=0.002)$ was significantly increased in T1DM patients. The absolute CD4-CD8TCRVa24 + iNKT cell number $(p=0.030)$ in T1DM patients was significantly lower than that in the HC group. Our findings suggested that the changes in the number of iNKT cells and their subsets in the peripheral blood of patients with type 1 diabetes may be one of the manifestations of the impaired immune system during the course of type 1 diabetes, and the immunoregulatory function may contribute the development of T1DM.

For the past few years, many studies have addressed the role of iNKT cells in T1DM. Evidence suggested that the number of iNKT cells in the thymus and spleen is significantly reduced in murine models and that the remaining iNKT cells are functionally deficient. In addition, stimulation of the remaining iNKT cells by a-GalCer inhibited the progression of T1DM, suggesting that iNKT cells exert protective effects on T1DM. However, data on the number and function of iNKT cells and their subpopulations in T1DM populations was inconsistent across several studies. Our findings suggested that the absolute iNKT cell number in the peripheral blood of type 1 diabetic patients was higher than that of normal healthy subjects $(p=0.03)$, which was consistent with the findings of the study by Oikawa et al., wherein the iNKT cells are overexpressed in the peripheral blood of newly diagnosed type 1 diabetic patients ${ }^{[18]}$. Some studies also showed a significant decrease in the number and function of iNKT cells in T1DM patients ${ }^{[19]}$. RomanGonzalez et al. evaluated the frequency and function of iNKT cells and their subsets in the peripheral blood of type 1 diabetic patients in the Colombian population and found that the frequency of iNKT cells and their subsets was similar in healthy controls and T1DM patients ${ }^{[20]}$. A similar result was observed in the study by Anna et al., wherein no difference was observed in the frequency of iNKT cells between healthy controls and T1DM patients ${ }^{[21]}$.

The changes in NKT subsets were inconclusive in the T1DM population. We found that the absolute number of CD $4+$ iNKT cells $(p<0.01)$, CD $8+i N K T$ cells $(p=0.021), C D 4+C D 8+i N K T$ cells $(p=0.002)$, and CD4-CD8-iNKT cells $(p=0.030)$ in patients with T1DM was significantly increased compared to healthy controls. Nevertheless, Kis et al. ${ }^{[23]}$ did not find any significant difference in the frequency of iNKT cells in the peripheral blood, and the number of CD $4+$ cells in iNKT cells was significantly decreased in patients with type 1 diabetes compared to that in the healthy groups. Montoya et al. ${ }^{[24]}$ found a significant increase in the frequency of CD4-CD8-iNKT cells in patients with T1DM compared to healthy controls. 
Several previous findings are not consistent with those in the current study. Herein, we analyzed the possible reasons: (1) Different identification methods of iNKT cells. The identification of iNKT cells relied on the combination of several monoclonal antibodies or CD1d tetramers. We defined iNKT cells with Va24, Vß11, and Va24Ja18 chain antibodies, respectively. Some studies defined iNKT cells as Vß11 fluorescein paired with Va24 antibody. (2) Patients differ in genetic background (white people or yellow people) and diabetes status (recent or long-term T1DM patients). The current study was conducted on yellow race, while the study of Roman-Gonzalez et al. was conducted on white race. Age may be a significant affecting factor. Peralbo et al. ${ }^{[25]}$ showed a decrease in the frequency of iNKT cells in healthy elderly, and aging might be related to a decrease in the frequency of peripheral blood iNKT cells. All the diabetes patients included in the current study were newly diagnosed T1DM patients, and the course of T1DM was not distinguished in some studies. In addition, the amount of iNKT varies markedly among individuals (from $0.01 \%$ to nearly $1 \%$ of peripheral lymphocytes) ${ }^{[26,27]}$, which might also lead to the detection error of the number of iNKT cells.

Several studies addressed the role of iNKT cells in adipose tissue, and their effect on body weight showed that iNKT cells might exert a protective role in weight gain and a catalytic role in weight loss; these findings were confirmed in both mouse and human studies. This phenomenon may be achieved through the role of iNKT cells in regulating the immune microenvironment and inhibiting the inflammatory response of adipose tissue ${ }^{[28-35]}$. iNKT cells may promote M2 type macrophage and inhibit M1 type macrophage differentiation by secreting IL-10 to alleviate inflammatory response in adipose tissue $\mathrm{e}^{[34,35]}$. In addition, iNKT cells regulate the secretion of adipocytes and inhibit the expression of leptin and adiponectin and the inflammatory reaction ${ }^{[34]}$. a-GalCerR specifically activates iNKT cells in adipose tissue, increases the expression of FGF21, increases heat production, increases energy expenditure, and finally reduces the weight of mice $e^{[35]}$.

Glycosylated hemoglobin is a continuous non-enzymatic reaction product between hemoglobin and hexoses (mainly glucose) in the blood during the erythrocyte lifetime. Since hemoglobin has a long halflife, it reflects an average blood glucose level ${ }^{[36]}$. The glycated hemoglobin level depends on the concentration and the action time of blood glucose. The current findings suggested that the absolute numbers in peripheral blood were significantly increased in T1DM patients and positively correlated with glycated hemoglobin. The high glycosylated hemoglobin at initial diagnosis indicates high blood glucose and duration of action, i.e., a severe condition at initial diagnosis. At least four different subsets exist in human iNKT cells according to the expression of CD4 and CD8 molecules. Four distinct subsets of iNKT cells were defined as CD4+, CD4-, CD4-CD8- (double negative), and CD4 + CD8+ (double-positive) ${ }^{[37]}$. It has been confirmed that CD4 + iNKT cells secrete Th2 cytokines (IL-4, IL-5, IL-10, and IL-13) ${ }^{[4]}$. These cytokines promote Th2 cell proliferation and inhibit Th1 cell proliferation, which might be the reason for the protective effect of iNKT cells in T1DM. The frequency of iNKT cells was lower in NOD mice than in non-autoimmune mice ${ }^{[38]}$. The repeated injection of a-GalCer protects NOD mice from the development of diabetes in several studies, while high levels of IL-4 and IL-10 have been detected in mice repeatedly injected with a-GalCer. Strikingly, CD4 + iNKT cells may exert a protective role in the pathogenesis of 
T1DM due to the secreted cytokines. Therefore, CD4 + iNKT cells may play a protective role in the course of T1DM and would be increased in newly diagnosed patients with type 1 diabetes and positively correlated with glycosylated hemoglobin, which might increase the reactivity after the destruction of the cells and the activation of autoimmunity leading to loss-of-function of the cells.

C-peptide is a 31-amino acid peptide produced in the secretory granules of pancreatic $b$ cells, as well as a polypeptide produced when proinsulin is lysed into insulin ${ }^{[39]}$. In healthy individuals, C-peptide and insulin are secreted in equal amounts into the blood. The C-peptide in circulation is characterized by slow clearance and long half-life, and hence, is considered as a biomarker of functional helper cells. The serum C-peptide level can be used to evaluate the islet function of patients and effectively grasp the synthesis and release of patients' islet cells ${ }^{[40]}$. The current results showed that the absolute CD $4+$ CD8 + iNKT number increased in newly diagnosed type 1 diabetic patients and was positively correlated with 30 and 60 min post-prandial C-peptide. Therefore, the role of CD4 + CD8 + iNKT cells in T1DM may be protective. However, little is known about CD4 + CD8 + iNKT cells, and the specific mechanism in autoimmunity needs further study. The proportion of CD4 + CD8 + iNKT cells in the total subpopulation of iNKT cells was small, and its role in the development process of T1DM may be limited. The development of type 1 diabetes involves complex interactions between the body's immune system and islet cells. However, the inflammation of islet is difficult to observe without histological evidence, and therefore, the analysis of cell components, such as NK cells, dendritic cells, and T cells, and the interactions between cells in peripheral blood may provide a basis for understanding the autoimmune status and the occurrence of diseases. In recent years, additional studies have shown that the iNKT cells play a crucial role in the development of autoimmune diseases. In this study, we investigated the changes in the number of iNKT cells and their subsets in the peripheral blood of newly diagnosed type 1 diabetic patients. The absolute iNKT cell number in the peripheral blood of T1DM patients was significantly higher than that of healthy subjects and was accompanied by changes in the number of cell subsets. The number of CD $4+$ iNKT cells increased in newly diagnosed patients with type 1 diabetes and were negatively correlated with $\mathrm{HbA} 1 \mathrm{c}$ and body mass index (BMI). The increase in CD4 + iNKT cells in the peripheral blood of T1DM patients may indicate an increase in the reactivity after the destruction of epithelial cells and the activation of autoimmunity leading to the loss of epithelial function. iNKT cells may play a role in fat metabolism and weight loss in T1DM patients, which could be exercised by CD $4+$ cell subsets. CD $4+$ CD8 + iNKT cells were increased in the peripheral blood of T1DM patients and were positively correlated with post-prandial C-peptide levels, suggesting that CD $4+C D 8+i N K T$ cells may exert a protective role in the pathogenesis of diabetes.

However, we only investigated the differences in the number of iNKT cells and their subsets between healthy people and type 1 diabetic patients but did not explore the mechanism of iNKT cells in the pathogenesis of T1DM. The analysis of the number of iNKT cells in type 1 diabetic patients is insufficient. Nevertheless, the current study has some limitations: small sample size and lack of longitudinal follow-up. We further investigated the role of iNKT cells and in the pathogenesis, as well as 
the potential diagnostic and prognostic value in patients with type 1 diabetes. Therefore, studies on the function of iNKT cells may provide new insights into the diagnosis and treatment of T1DM.

\section{Declarations}

\section{Funding}

No funding was received.

\section{Conflicts of interest/Competing interests}

The authors declared that they have no conflicts of interest to this work. We declare that we do not have any commercial or associative interest that represents a conflict of interest in connection with the work submitted

\section{Availability of data and material}

The datasets used or analysed during the current study are available from the corresponding author on reasonable request.

\section{Code availability}

Not applicable

\section{Authors' contributions}

Shuci Tian: Data collection and manuscript writing.

Lichao Gao: Conception and design, data collection and manuscript revision. Contributed equally to this study, share first authorship.

Hui Guo: Conception, revision and final approval of the version.

\section{References}

1. M. Kronenberg, Toward an understanding of NKT cell biology: progress and paradoxes[J]. Annu. Rev. Immunol. 23, 877-900 (2005)

2. Laurent Gapin, Development of invariant natural killer T cells[J]. Curr. Opin. Immunol. 9, 68-74 (2016)

3. C. Susannah, J. Shissler, Tonya, Webb, The Ins and Outs of Type I iNKT Cell Development[J]. Mol Immunol 105, 116-130 (2019)

4. P. Dellabona, E. Padovan, G. Casorati et al., An invariant Va24-JaQ/V 111 T cell receptor is expressed in all individuals by clonally expanded CD4-8- T cells[J]. J. Exp. Med. 180(3), 1171-1176 (1994) 
5. L. Van Kaer, V.V. Parekh, L. Wu, Invariant natural killer T cells: bridging innate and adaptive immunity[J]. Cell Tissue Res 343, 43-55 (2011)

6. L. Van Kaer, V.V. Parekh, L. Wu, The response of CD1d-restricted invariant NKT cells to microbial pathogens and their products[J]. Front. Immunol. 6, 226 (2015)

7. M.P. Morran, A. Vonberg, A. Khadra et al., Immunogenetics of Type 1 Diabetes Mellitus[J]. Mol Aspects Med 42, 42-60 (2015)

8. B. Lisa Kain, L. Webb, Brian, Anderson, The Identification of the Endogenous Ligands of Natural Killer T Cells Reveals the Presence of Mammalian a-Linked Glycosylceramides[J]. Immunity 41(4), 543554 (2014)

9. M. McDufile, N.A. Maybee, S.R. Keller, et aL. Nonobese diabetic (NOD)mice congenic for a targeted deletion of 12/15 lipoxygen-ase are protected from autoimmune diabetes[J].Diabetes, 2008, 57(1): 199-208

10. S. Sharif, G.A. Arreaza, P. Zucker et al., Activation of natural killer T cells by alpha-galactosylceramide treatment prevents the onset and recurrence of autoimmune type 1 diabetes. Nat Med 7, 1057-1062 (2001)

11. S. Hong, M.T. Wilson, I. Serizawa, L. Wu et al., The natural killer T-cell ligand alphagalactosylceramide prevents autoimmune diabetes in non-obese diabetic mice[J]. Nat Med 7, 10521056 (2001)

12. S. Sharif, G.A. Arreaza, P. Zucker et al., Activation of natural killer T cells by alpha-galactosylceramide treatment prevents the onset and recurrence of autoimmune type 1 diabetes. Nat Med 7, 1057-1062 (2001)

13. W. Li, F. Ji, Y. Zhang et al., Cooperation of invariant NKT cells and CD $4+$ CD25 + T regulatory cells in prevention of autoimmune diabetes in non-obese diabetic mice treated with a-galactosylceramide[J]. Acta Biochim Biophys Sin (Shanghai),2008, 40(5): 381-390

14. S. Hong, M.T. Wilson et al., The natural killer T-cell ligand a-galactosylceramide prevents autoimmune diabetes in non-obese diabetic mice. Nat.Med 7, 1052-1056 (2001)

15. T. Claire Forestier, A. Takaki, Molano et al., Improved Outcomes in NOD Mice Treated with a Novel Th2 Cytokine-Biasing. NKT Cell Activator[J].J Immunol 173(3), 1415-1425 (2007)

16. D.V. Baev, S. Caielli, F. Ronchi et al., Impaired SLAM-SLAM Homotypic Interaction between Invariant NKT Cells and Dendritic Cells Affects Differentiation of IL-4/IL-10-Secreting NKT2 Cells in Nonobese Diabetic Mice[J]. The Journal of immunology 181(2), 868-877 (2008)

17. J.M. Coquet, K. Kyparissoudis, D.G. Pellicci et al., IL-21 Is Produced by NKT Cells and Modulates NKT Cell Activation and Cytokine Production[J]. J. Immunol. 178, 2827-2834 (2007)

18. A. Yoichi Oikawa, S. Shimada, Yamada et al., High Frequency of valpha24(+) vbeta11(+) T-cells Observed in Type 1 Diabetes[J]. Diabetes Care 25(10), 1818-1823 (2002)

19. Y.A.N.G. Ming-lan, ZHANG Xiao-fang, WANG Li, et al. Changes of natural killer cells in peripheral blood in patients with newly-diagnosed type 1 diabetes mellitus[J]. Chinese Journal of Endocrinology and Metabolism, 2012, 28(6): 455-459 
20. A.Roman-Gonzalez,M.Eugenia Moreno, J.M. Alfaro et al., Frequency and Function of Circulating Invariant NKT Cells in Autoimmune Diabetes Mellitus and Thyroid Diseases in Colombian Patients[J]. Hum. Immunol. 70(4), 262-268 (2009)

21. S. Anna, S. Tocheva, G.H. Mansour, Tristan, Holt, The Clonal Invariant NKT Cell Repertoire in People with Type 1 Diabetes Is Characterized by a Loss of Clones Expressing High-Affinity TCRs[J]. J. Immunol. 198(4), 1452-1459 (2017)

22. Nonantzin Beristain-Covarrubias, Elsy Canche-Pool, † Rita Gomez-Diaz, et al. Reduced iNKT Cells Numbers in Type 1 Diabetes Patients and Their First-Degree Relatives[J].Immun Inflamm Dis, 2015, 3 (4): $411-419$

23. J. Kis, P. Engelmann, K. Farkas et al., Reduced CD4 + Subset and Th1 Bias of the Human iNKT Cells in Type 1 Diabetes Mellitus[J]. J Leukoc Biol 81(3), 654-662 (2007)

24. J. Carlos, D. Montoya, J. Pollard, Martinson et al., Characterization of Human Invariant Natural Killer T Subsets in Health and Disease Using a Novel Invariant Natural Killer T Cell-Clonotypic Monoclonal Antibody, 6B11[J]. Immunology 122(1), 1-14 (2007)

25. O. Esther Peralbo, I. DelaRosa, Gayoso et al., Decreased Frequency and Proliferative Response of Invariant Valpha24Vbeta11 Natural Killer T (iNKT) Cells in Healthy Elderly[J]. Biogerontology 7(5-6), 483-492 (2006)

26. P.T. Lee, K. Benlagha, L. Teyton, A. Bendelac, Distinct functional lineages of human V(alpha)24 natural killer T cells[J]. J. Exp. Med. 195(5), 637-641 (2002)

27. S.P. Berzins, M.J. Smyth, A.G. Baxter, Presumed guilty: natural killer T cell defects and human disease[J]. Nat Rev Immuno 11(2), 131-142 (2011)

28. L. Lynch, M. Nowak, B. Varghese,et al, Adipose tissue invariant NKT cells protect against diet induced obesity and metabolic disorder through regulatory cytokine production[J]. Immunity $37(3), 574-587$ (2012)

29. L. Lynch, D. O'Shea, D.C. Winter,et al, Invariant NKT cells and CD1d(+) cells amass in human omentum and are depleted in patients with cancer and obesity[J]. Eur J Immunol 39(7), 1893-1901 (2009)

30. A.M. Daniela Strodthoff, H.E. Lundberg, Agardh ,et al, Lack of Invariant Natural Killer T Cells Affects Lipid Metabolism in Adipose Tissue of Diet-Induced, Arteriosclerosis, Thrombosis, and Vascular[J]. Biology 33, 1189-1196 (2013)

31. Soledad, López, Sara García-Serrano, Carolina Gutierrez-Repiso, et al. Tissue-Specific Phenotype and Activation of iNKT Cells in Morbidly Obese Subjects: Interaction With Adipocytes and Effect of Bariatric Surgery[J]. Obes Surg, 2018, 28 (9): 2774-2782

32. M.M. Tiemessen, A.L. Jagger, H.G. Evans et al., CD4 + CD25 + FOXP3 + regulatory T cells induce alternative activation of human monocytes/macrophages[J]. Proc. Natl. Acad. Sci. U.S.A. 104(49), 19446-19451 (2007)

33. Zhu Xu Hanyuan, Gong Huijuan, Fengying, Invariant natural killer T cells and obesity[J]. International Journal of Endocrinology and Metabolism, 2019, 39(1): 40-44 
34. F. Sirico, A. Bianco, G. D'Alicandro et al., Effects of physical exercise on adiponectin, leptin, and inflammatory markers in childhood obesity: systematic review and meta analysis[J]. Child Obes 14(4), 207-217 (2018)

35. A.E. Lydia Lynch, D.D. Hogan, iNKT Cells Induce FGF21 for Thermogenesis and Are Required for Maximal Weight Loss in GLP1 Therapy[J]. Cell Metab. 24(3), 510-519 (2016)

36. Yang Jie, Diagnosis value of combined test of glycosylated hemoglobin and serum $C$ peptide on diagnosis diabetes[J]. Chinese Journal of Practical Medicine 45(18), 31-32, 36 (2018)

37. C.J. Montoya, D. Pollard, J. Martinson et al., Characterization of human invariant natural killer T subsets in health and disease using a novel invariant natural killer T cell-clonotypic monoclonal antibody, 6B11[J]. Immunology 122(1), 1-14 (2007)

38. Y. Liana Ghazarian, Simoni, Invariant NKT cell development: focus on NOD mice[J]. Curr. Opin. Immunol. 27, 83-88 (2014)

39. D.F. Steiner, D. Cunningham, L. Spigelman et al., Insulin biosynthesis: evidence for a precursor[J]. Science 157, 697-700 (1967)

40. J. Wahren, A. Kallas, A.A. Sima, The clinical potential of C-peptide replacement in 943 type 1 diabetes[J]. Diabetes 61, 761-772 (2012)

\section{Figures}




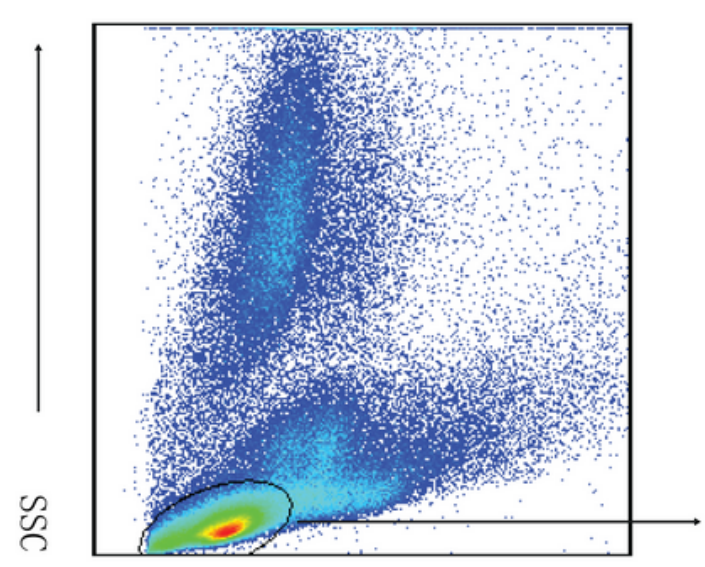

FSC
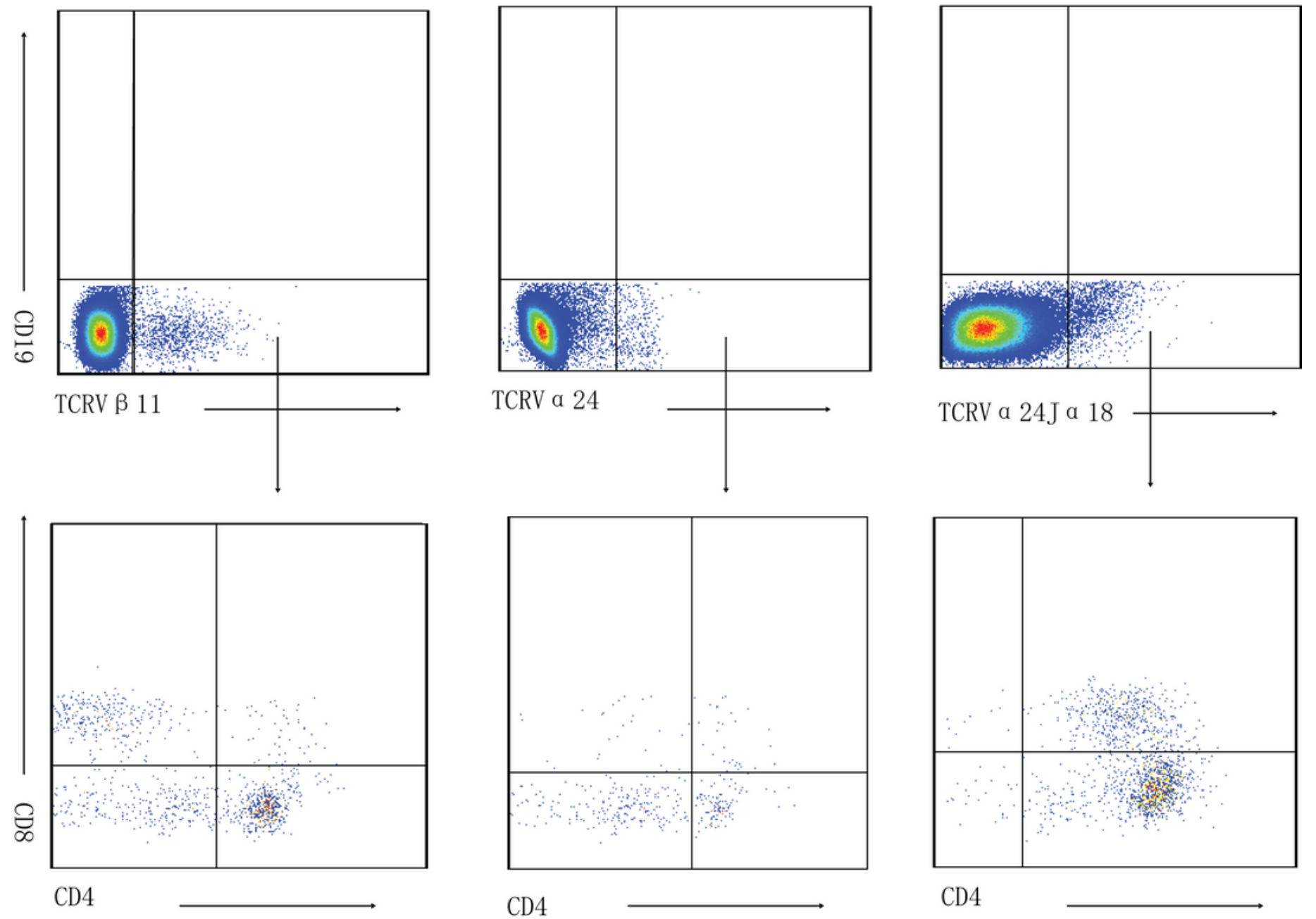

Figure 1

flow cytometry analysis of two groups of human peripheral blood iNKT cells and their subsets 

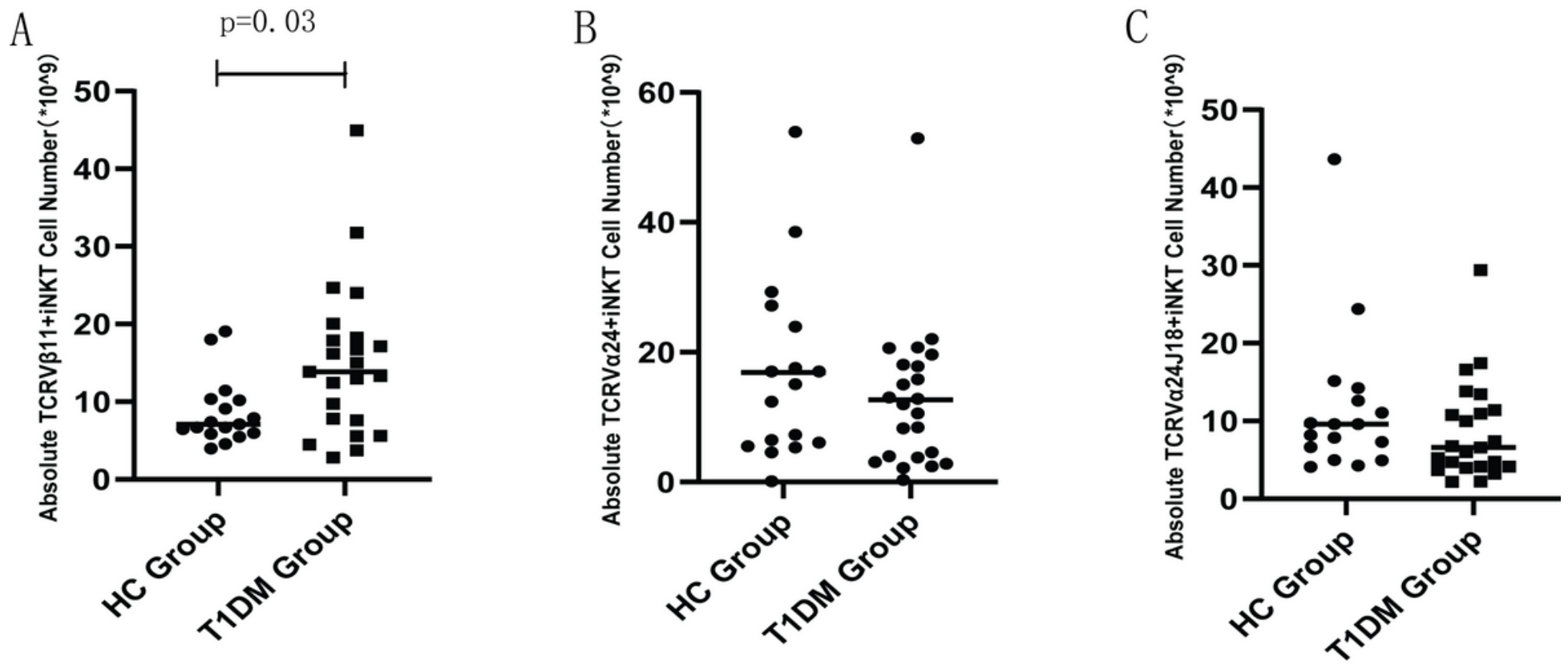

Figure 2

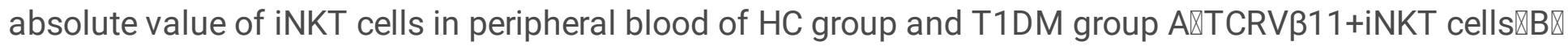
TCRVa24+iNKT cells $囚 \mathrm{C} \otimes T C R V a 24$ Ja18+iNKT cells. 
A
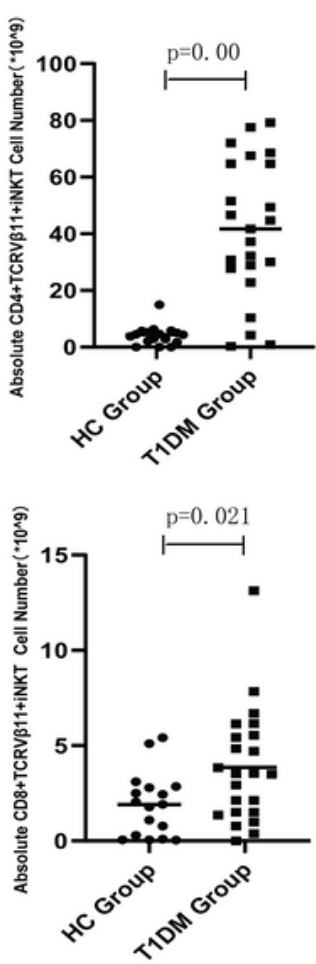

C

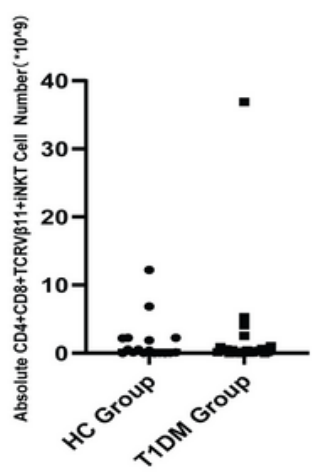

D

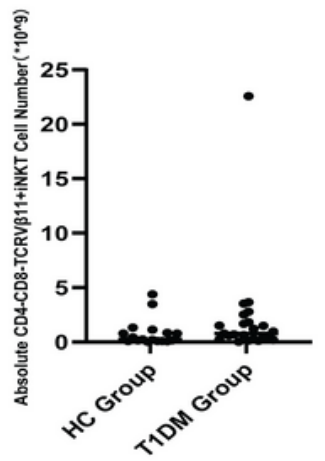

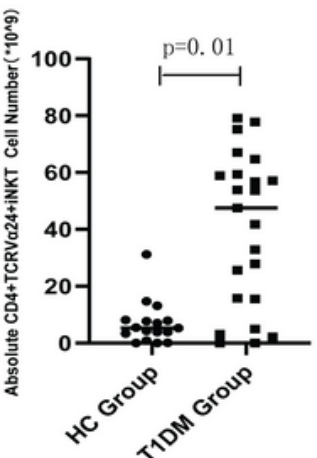
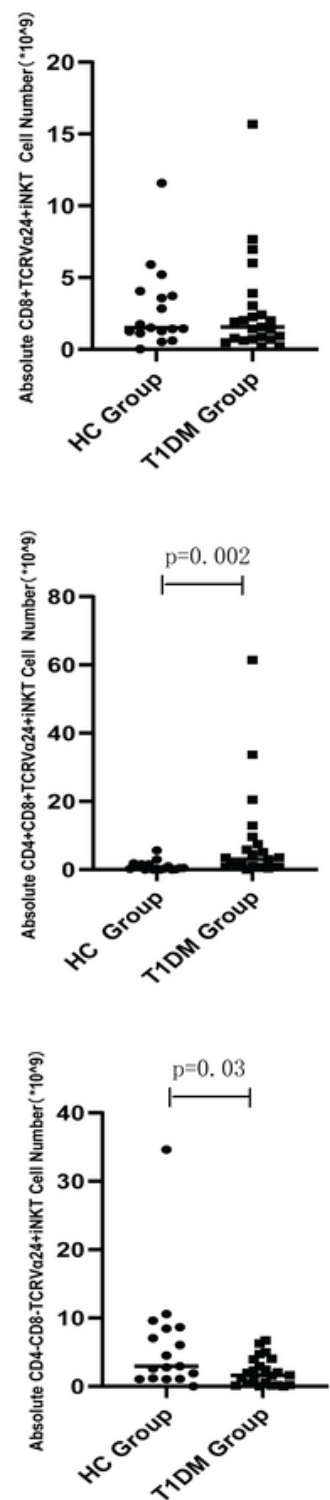
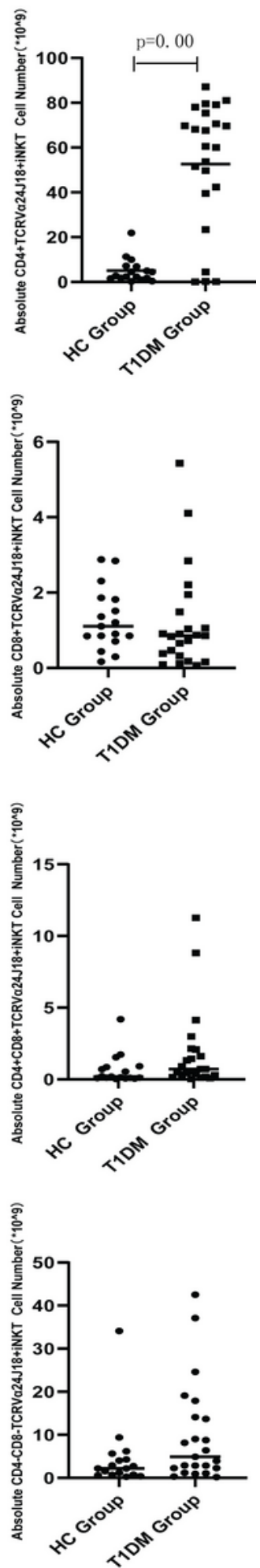

Figure 3

absolute value of subsets of iNKT cells in peripheral blood of HC group and T1DM group A $\triangle$ CD4+iNKTcells $₫ B \otimes C D 8+i N K T$ cells $₫ C \otimes C D 4+C D 8+i N K T$ cells $₫ D \otimes C D 4-C D 8-i N K T$ cells. 

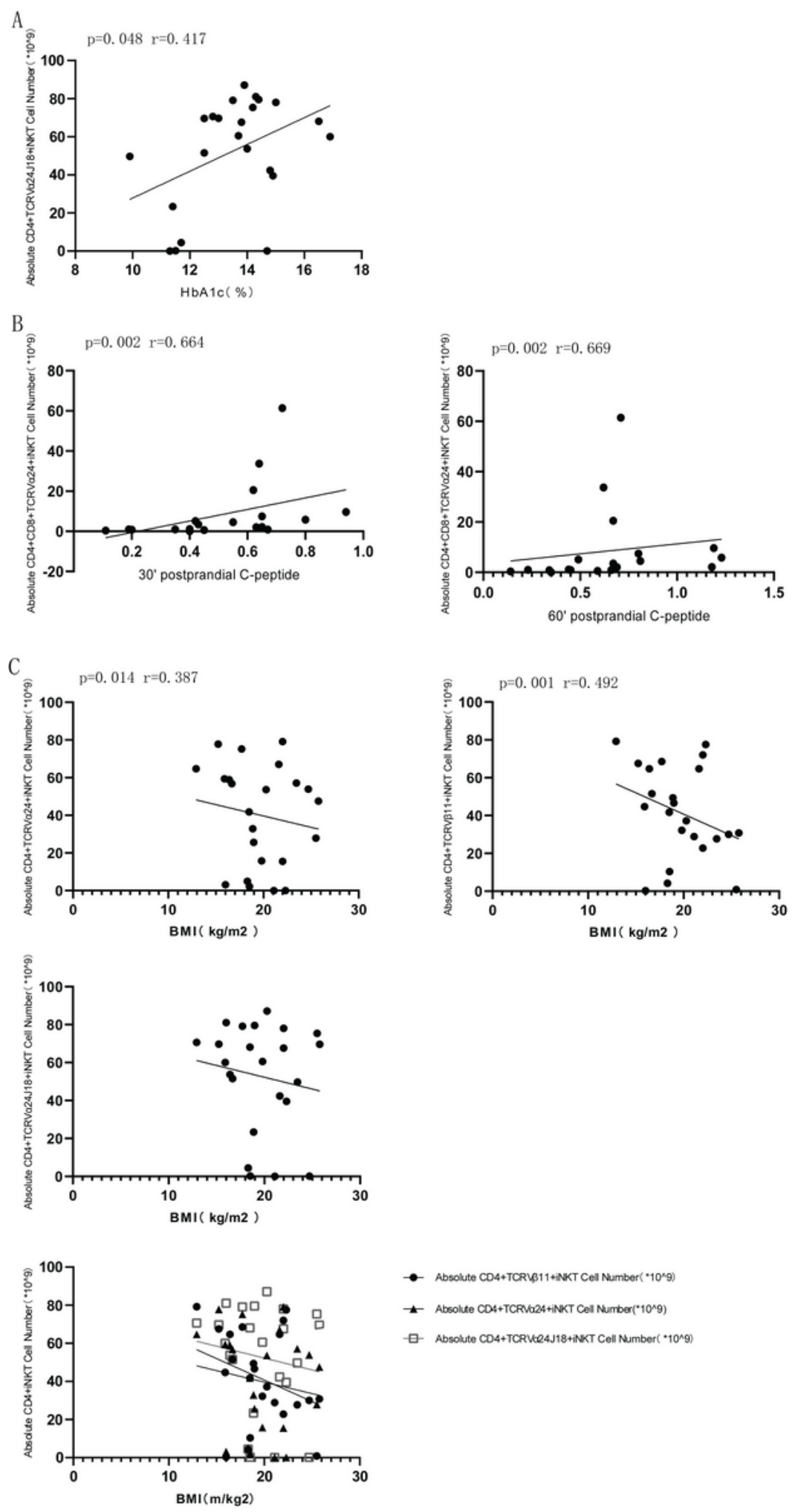

\section{Figure 4}

Correlation between absolute value of subsets of iNKT cells and clinical indicators A: Correlation between absolute value of subsets of iNKT cells and $\mathrm{HbA} 1 \mathrm{c} \%$; $\mathrm{B}$ : Correlation between absolute value of subsets of iNKT cells and $\mathrm{C}$ peptide; $\mathrm{C}$ : Correlation between absolute value of subsets of iNKT cells and BM 